\title{
Two-sided weighted Fourier inequalities
}

\author{
ELIJAH LifLyAND AND SERGEY TIKHONOV
}

\begin{abstract}
Fourier transform estimates for $\|\widehat{f}\|_{L_{q, \widetilde{w}}}$ via $\|f\|_{L_{p, w}}$ from above and from below are studied. For $p=q$, equivalence results, i.e.,

$C_{1}\|f\|_{L_{p, w}} \leq\|\widehat{f}\|_{L_{p, \widetilde{w}}} \leq C_{2}\|f\|_{L_{p, w}}, \quad \widetilde{w}(x)=w(1 / x) x^{p-2}, \quad 1 \leq p<\infty$, are shown to be valid for functions from certain classes under the Muckenhoupt conditions: $w \in A_{p}$ or $w \in A_{2 p}$. Sharpness of these conditions is proved.
\end{abstract}

Mathematics Subject Classification (2010): 42A38 (primary); 26D15, 46E30 (secondary).

\section{Introduction}

The theory of Weighted Fourier Inequalities studies the following $\left(L_{p}, L_{q}\right)$ inequalities:

$$
\|\widehat{f}\|_{L_{q, \widetilde{w}}(\mathbb{R})} \lesssim\|f\|_{L_{p, w}(\mathbb{R})}, \quad 1 \leq p \leq q<\infty,
$$

where the Fourier transform

$$
\widehat{f}(x)=\int_{\mathbb{R}} f(t) e^{-i x t} d t
$$

is defined in certain regular or distributional sense (see, e.g., [8]).

We use the notation " $\lesssim$ " and " $\gtrsim$ " as abbreviations for " $\leq C$ " and " $\geq C$ ", respectively, while "こ” means that " $\lesssim$ " and " $\gtrsim$ " hold simultaneously. Here and below $C, C_{1}, C_{2}$ denote positive constants.

The norms in weighted spaces are defined by

$$
\|f\|_{L_{p, w}(\mathbb{R})}=\left(\int_{\mathbb{R}}|f(x)|^{p} w(x) d x\right)^{1 / p}
$$

The research was supported, in part, by grants 2009 SGR 1303, MTM2008-05561-C02-02/MTM, RFFI 09-01-00175, and the ESF Network Program HCAA.

Received May 30, 2010; accepted in revised form December 17, 2010. 
for $L_{p, w}$, and similarly for $L_{q}, \widetilde{w}$, where $w$ and $\widetilde{w}$ are weights, that is, non-negative, locally integrable functions on $\mathbb{R}$.

Such inequalities are not only of interest by themselves but also have important applications (this is discussed in [8]), such as the uncertainty principle [3], and restriction theory (see, e.g., [5,9]). In a simple form, one faces such inequalities while justifying the differentiation formula for Fourier transforms.

The celebrated Pitt's theorem (see, e.g., [7]; in [27,33] the case of Fourier series is considered) states that for the power weights

$$
w(x)=|x|^{\gamma} \text { and } \tilde{w}=|x|^{-\gamma q / p+q / p^{\prime}-1},
$$

(1.1) holds for all $f \in L_{p, w}$ if and only if

$$
\gamma \in[\max \{0, p-2\}, p-1) \text {. }
$$

In particular, $\widehat{f}$ is well-defined in this case (see [4,6], [7, Example 5]).

In fact, Pitt's theorem contains the Plancherel theorem and classical (nonweighted) versions of both Hardy-Littlewood's inequality $(1<p=q \leq 2, \gamma=0$ or $p=q \geq 2, \gamma=p-2$; see [36, Theorem 79, 80]) and Hausdorff-Young's inequality $\left(q=p^{\prime} \geq 2, \gamma=0\right.$; see [36, Theorem 74]).

A natural question arises of whether the results of type $(\mathcal{P})$ can be obtained with a larger selection of weights. Our first observation is concerned with monotone weights. In general, sufficient conditions in terms of weights were studied in [4$7,18,21,22,26]$, etc. Typically, conditions for (1.1) to hold are given in terms of decreasing rearrangements of weights. Moreover, when the weights are assumed monotone, inequality (1.1) necessarily implies certain integral conditions in terms of $w$ and $\widetilde{w}$. To formulate such results due to Benedetto et al. [6], we recall the Muckenhoupt $A_{p}$ condition (see, e.g., [34, Chapter V]).

For $1<p<\infty, w \in A_{p}(\mathbb{R})$ if

$$
\left(\frac{1}{|I|} \int_{I} w(x) d x\right)\left(\frac{1}{|I|} \int_{I} w(x)^{-1 /(p-1)} d x\right)^{p-1} \lesssim 1
$$

for all intervals $I \subset \mathbb{R}$. For $p=1, w \in A_{1}(\mathbb{R})$ if

$$
\frac{1}{|I|} \int_{I} w(t) d t \lesssim w(x)
$$

for almost all $x \in I$ and all intervals $I \subset \mathbb{R}$.

The following generalization of $(\mathcal{P})$ provides the necessary and sufficient condition for (1.1) to hold for monotone increasing weights ([6]). It is an analogue of the condition $\gamma \geq 0$ in Pitt's inequality.

Theorem A. Let $1<p \leq q \leq p^{\prime}<\infty$ and $w$ be an even weight which is non-decreasing on $\mathbb{R}_{+}$. Then for $\widetilde{w}(x):=w(1 / x)^{q / p}|x|^{q / p^{\prime}-1}$,

$$
\text { (1.1) holds if and only if } w^{q / p} \in A_{1+\left(q / p^{\prime}\right)}(\mathbb{R}) \text {, }
$$

or, equivalently, if and only if $w \in A_{p}(\mathbb{R})$. 
We note that, in general, for any weight $w$ and $\alpha \geq 1$,

$$
w^{\alpha} \in A_{1+\alpha(p-1)}(\mathbb{R}) \quad \Longrightarrow \quad w \in A_{p}(\mathbb{R}),
$$

which easily follows from the monotonicity in $\alpha$ of the integral means $\left(\frac{1}{|I|} \int_{I} w(x)^{\alpha} d x\right)^{1 / \alpha}$. On the other hand, using [14, Proposition 2.3(d)] (see also [15, Corollary 6.3]), we get the reverse implication in (1.4) for any even nondecreasing weight $w$. Taking $\alpha=q / p$ gives an equivalence of the two Muckenhoupt's conditions in Theorem A.

We also remark that assuming $w$ to be non-decreasing is essential, since, for $p=q$ say, (1.1) with any non-increasing weight $w \neq 0$ implies $w \asymp$ const (see [6, Section 4]).

Let us now refer to functions rather than to weights. Our second observation is related to functions which are either monotone or general monotone; the latter will be specified below (see (1.6)). It is convenient to study such functions on $\mathbb{R}_{+}$. Further we use

$$
\|f\|_{p, w}=\left(\int_{\mathbb{R}_{+}}|f(x)|^{p} w(x) d x\right)^{1 / p}, \quad \widehat{f}_{+}(x)=\int_{\mathbb{R}_{+}} f(t) e^{-i x t} d t,
$$

and

$$
\widehat{f}_{\cos }(x)=\int_{\mathbb{R}_{+}} f(t) \cos x t d t, \quad \widehat{f}_{\sin }(x)=\int_{\mathbb{R}_{+}} f(t) \sin x t d t,
$$

with obvious relation $\widehat{f}_{+}=\widehat{f}_{\cos }-i \widehat{f}_{\text {sin }}$. We will assume $w$ to be even: in this case $w \in A_{p}(\mathbb{R})$ if and only if $w \in A_{p}\left(\mathbb{R}_{+}\right)$, and $\left\|\widehat{f}_{\cos }\right\|_{p, w} \asymp\|\widehat{f}\|_{L_{p, w}(\mathbb{R})}$ when $f$ is even, while $\left\|\widehat{f}_{\sin }\right\|_{p, w} \asymp\|\widehat{f}\|_{L_{p, w}(\mathbb{R})}$ when $f$ is odd.

The well-known Hardy-Littlewood theorem [36, Chapter IV] states that

$$
\|\widehat{f}\|_{p, \widetilde{w}} \asymp\|f\|_{p, w}, \quad \widetilde{w}(x):=w(1 / x) x^{p-2},
$$

with $\widehat{f}=\widehat{f_{\text {cos }}}, 1<p<\infty$, and $w \equiv$ const, holds true for any monotone decreasing function $f$ integrable near zero.

The weighted - for power weights - version of (1.5) came as a conjecture of Boas in [10]; the proof followed shortly after: Sagher [29]. We present that statement as

Theorem B. Let $1<p<\infty$ and $w(t)=t^{\gamma}$. Then for any monotone decreasing function $f$, integrable on $[0,1]$,

(1.5) holds if $-1<\gamma<p-1$. 
Comparing now $(\mathcal{B})$ and $(\mathcal{P})$, we note that monotonicity of functions, on the one hand, extends the range for $\gamma$ in Pitt's inequality and, on the other hand, allows one to write a two-sided inequality. The study of both of these phenomena for more general function classes and for weights subject to a Muckenhoupt condition is the main goal of this paper. Our key result (see Theorem 3.1) states that for $w \in A_{p}$, equivalence (1.5) holds for each general monotone function, that is (see [23]), for $f: \mathbb{R}_{+} \rightarrow \mathbb{R}_{+}$locally of bounded variation out of the origin and vanishing at infinity (we will call such functions admissible), which satisfies for any $x>0$

$$
\int_{x}^{2 x}|d f(t)| \leq C \int_{x / c}^{c x} \frac{|f(t)|}{t} d t,
$$

with some $c>1$. If an admissible $f$ satisfies (1.6), we will write $f \in G M$.

Assuming additionally the weight $w$ be such that $w(x) \asymp w(y)$ for any $y \leq$ $x \leq 2 y$ (written $w \in \Delta_{2}^{2}$ ), we can obtain the following extension of Theorem $\overline{\mathrm{B}}$ (see Theorem 3.3): Equivalence (1.5) holds

(A) for any general monotone $f$ and for $\left(f, \widehat{f}_{\mathrm{cos}}\right)$ if and only if $w \in A_{p}\left(\mathbb{R}_{+}\right)$, $1<p<\infty$

$$
\text { or } \int_{\delta}^{\infty} \frac{w(x)}{x} d x \lesssim w(\delta), \quad p=1 \text {; }
$$

(B) for any general monotone $f$ and for $\left(f, \widehat{f}_{\sin }\right)$ if and only if $w \in A_{2 p}\left(\mathbb{R}_{+}\right)$, $1 \leq p<\infty$.

For the power weights $w(x)=x^{\gamma}$ it just means that $(\mathcal{B})$ is refined as follows: (1.5) holds true for $\left(f, \widehat{f}_{\cos }\right)$, with $f \in G M$, if and only if $\gamma \in(-1, p-1)$, while for $\left(f, \widehat{f}_{\text {sin }}\right)$, with $f \in G M$, if and only if $\gamma \in(-1,2 p-1)$.

We remind the reader that the range $\max \{0, p-2\} \leq \gamma<p-1$ is, generally, sharp for Pitt's inequality $\|\widehat{f}\|_{p, \widetilde{w}} \lesssim\|f\|_{p, w}$ to hold without any regularity assumption on $f$. Attempts to widen this range were taken by many authors (see, e.g., $[5,8,16,17,28,31])$. A typical condition for this is vanishing of certain moments of function $f$. In particular, Sadosky and Wheeden [28] proved that the $\left(L_{p}, L_{p}\right)$ Pitt's inequality holds for $p-1<\gamma<2 p-1$ for $f$ having mean value zero. Unlike the approach for general monotone functions, the limiting case $\gamma=p-1$ is excluded even under this restriction on $f$.

The paper is organized as follows. We start with $L_{1}$ estimates in Section 2. In Section 3, we formulate our main results. In Section 4 we prove general upper and lower estimates for the integrability with weights of the Fourier transforms. In particular, we extend Theorem A by assuming either regularity of $w$ or regularity of $f$ :

Theorem 1.1. Let $1<p \leq q<\infty$ and an even weight $w^{q / p} \in A_{1+\left(q / p^{\prime}\right)}(\mathbb{R})$. Then Pitt's inequality $\|\widehat{f}\|_{q, \widetilde{w}} \lesssim\|f\|_{p, w}$ holds provided either $w$ is increasing $\left(q \leq p^{\prime}\right)$, or $f$ is general monotone. 
In the last section we encompass multidimensional generalizations of Boas type result $(\mathcal{B})$ for radial functions.

We finally mention that the class of general monotone functions consists of functions with much more complicated structure than monotone ones [25]. It is clear that any monotone function is in $G M$. Further, a function $f$ on $\mathbb{R}_{+}$is quasimonotone if there exists $\tau>0$ such that $f(x) / x^{\tau}$ is monotone decreasing. Such functions are in $G M$ as well. However, for any monotone $f$ one can define

$$
\begin{aligned}
f_{1}(x) & :=f(x) \chi_{(0,1]}(x)+\sum_{n=0}^{\infty} a_{n} f(x) \chi_{\left(\lambda^{n}, \lambda^{n+1}\right]}(x), \quad \lambda>1, \\
a_{n} & =\left\{\begin{array}{l}
a_{2 k}=1, \\
a_{2 k+1}=0,
\end{array} k=0,1,2, \cdots\right.
\end{aligned}
$$

so that $f_{1}$ is general monotone but neither monotone nor quasi-monotone. Note that if we consider a function with different oscillation properties, say

$$
f_{2}(x):=f_{2}(f, x):=\sum_{n=0}^{\infty} a_{n} f(x) \chi_{(n, n+1]}(x),
$$

then $f_{2} \in G M$ not for all $f$. For example, if $f(x)=x^{-l}, l \in \mathbb{R}$, then $f_{2} \notin G M$, while if $f(x)=e^{-x}$, then $f_{2} \in G M$.

One of the properties of the $G M$ functions often used is (see [25])

$$
|f(x)| \lesssim \int_{x / c}^{c x} \frac{|f(t)|}{t} d t, \quad c>1
$$

ACKNOWLEDGEMEnTs. The authors are indebted to Fulvio Ricci for stimulating discussions. The authors gratefully acknowledge the support of the Centre de Recerca Matemàtica in Barcelona and the Centro di Ricerca Matematica Ennio De Giorgi in Pisa.

\section{Starting points: $L_{1}$ estimates}

In this section, weighted Fourier inequalities are studied in $L_{1}$. In particular, we give pointwise estimates from above of the Fourier transforms (see Lemma 2.2) and lower estimates for the integrated Fourier transforms (see Lemma 2.3). These auxiliary results will be useful in our further study. Using Lemmas 2.2 and 2.3, we estimate

$$
\int_{\mathbb{R}_{+}}\left|\widehat{f}_{\cos }(x)\right| x^{-1} w(1 / x) d x \text { and } \int_{\mathbb{R}_{+}}\left|\widehat{f}_{\sin }(x)\right| x^{-1} w(1 / x) d x
$$


from above and from below (see Propositions 2.1 and 2.4). Denoting

$$
\Phi(t)=\Phi(f ; t)=\int_{t}^{2 t}|d f(s)|
$$

we first give upper estimates.

Proposition 2.1. Let $f$ be admissible. If $f$ is integrable near the origin,

$$
\int_{0}^{\infty} x^{-1} w\left(x^{-1}\right)\left|\widehat{f}_{\cos }(x)\right| d x \lesssim \int_{0}^{\infty}|\Phi(t)|\left[t^{-1} \int_{0}^{t} w(x) d x+\int_{t}^{\infty} x^{-1} w(x) d x\right] d t .
$$

If $f(t)$ is integrable near the origin,

$$
\int_{0}^{\infty} x^{-1} w\left(x^{-1}\right)\left|\widehat{f}_{\text {sin }}(x)\right| d x \lesssim \int_{0}^{\infty}|\Phi(t)|\left[t^{-1} \int_{0}^{t} w(x) d x+t \int_{t}^{\infty} x^{-2} w(x) d x\right] d t .
$$

Proof. The following auxiliary result was proved in [23] (see Lemma 2.2 and corresponding remark on page 1141).

Lemma 2.2. Let $f$ be admissible. Then

$$
\left|\widehat{f}_{\cos }(x)\right| \lesssim \int_{0}^{1 / x} \Phi(t) d t+x^{-1} \int_{1 / x}^{\infty} t^{-1} \Phi(t) d t
$$

and

$$
\left|\widehat{f}_{\sin }(x)\right| \lesssim x \int_{0}^{1 / x} t \Phi(t) d t+x^{-1} \int_{1 / x}^{\infty} t^{-1} \Phi(t) d t .
$$

The proof of Proposition 2.1 immediately follows from Lemma 2.2 and Fubini's theorem.

To obtain general lower estimates for $p=1$, we need a preliminary result that will be used for lower estimates when $p>1$ as well.

Lemma 2.3. Let $\widehat{f}_{\cos }$ and $\widehat{f}_{\sin }$ exist as improper integrals, converge uniformly on every compact set away from zero, and be locally Lebesgue integrable over $\mathbb{R}_{+}$. Let $f \geq 0$ such that

$$
\int_{1}^{\infty} \frac{f(t)}{t} d t<\infty
$$

and $c>1$. If $f(t)$ is integrable near zero, we have

$$
\int_{z / c}^{c z} t^{-1} f(t) d t \lesssim \int_{0}^{1 / z}\left|\widehat{f}_{\cos }(x)\right| d x,
$$

and if $t f(t)$ is integrable near zero, we have

$$
\int_{z / c}^{c z} t^{-1} f(t) d t \lesssim \int_{0}^{1 / z}\left|\widehat{f}_{\sin }(x)\right| d x .
$$


Proof of Lemma 2.3. Let, for example, $\widehat{f}_{\text {sin }}$ be subject to the same assumptions as in the lemma. Since we understand the Fourier transform in the improper sense, we have for $x \geq \varepsilon, \varepsilon>0$ fixed, and $A$ large enough $\int_{0}^{A} f(t) \sin x t d t=\widehat{f}_{\sin }(x)+o(1)$. By this, on each finite interval $[\varepsilon, u]$ the family $\int_{0}^{A} f(t) \sin x t d t$ is controlled by the integrable function $\left|\widehat{f}_{\text {sin }}(x)\right|+1$. For $f \geq 0$, applying the Lebesgue dominated convergence theorem, we have

$$
\int_{\varepsilon}^{u} \widehat{f_{\sin }}(x) d x=\lim _{A \rightarrow \infty} \int_{\varepsilon}^{u} \int_{0}^{A} f(t) \sin x t d t d x=\int_{0}^{\infty} f(t) \frac{\cos \varepsilon t-\cos u t}{t} d t .
$$

Using assumptions on $f$ and dominated convergence, we obtain, letting now $\varepsilon$ tend to zero,

$$
\int_{0}^{u} \widehat{f}_{\sin }(x) d x=2 \int_{0}^{\infty} f(t) t^{-1} \sin ^{2}(u t / 2) d t \geq 0 .
$$

Further,

$$
\begin{aligned}
\int_{0}^{1 / z}\left|\widehat{f}_{\sin }(x)\right| d x & \geq \int_{0}^{1 / c z}\left|\widehat{f}_{\sin }(x)\right| d x \geq\left|\int_{0}^{1 / c z} \widehat{f}_{\sin }(x) d x\right| \\
& =2 \int_{0}^{\infty} f(t) t^{-1} \sin ^{2}(t /(2 c z)) d t \\
& \geq 2 \sin ^{2}\left(1 / 2 c^{2}\right) \int_{z / c}^{c z} t^{-1} f(t) d t \geq 0,
\end{aligned}
$$

the desired estimate. For $\widehat{f}_{\mathrm{cos}}$, the proof is similar for

$$
\int_{0}^{u} \widehat{f}_{\cos }(x)(1-x) d x .
$$

A slightly different argument for integrable $f$ can be found in [21]. The proof is complete.

Let, for some $c>1$,

$$
\Psi(x)=\Psi(f ; x)=\int_{x / c}^{c x} t^{-1} f(t) d t .
$$

Proposition 2.4. Let $f \geq 0$ satisfy (2.6). If $f \in L_{1}(0,1)$, then

$$
\int_{0}^{\infty} \Psi(x) w(x) d x \lesssim \int_{0}^{\infty}\left|\widehat{f}_{\cos }(t)\right|\left(\int_{0}^{1 / t} w(x) d x\right) d t ;
$$

and if $t f(t) \in L_{1}(0,1)$, then

$$
\int_{0}^{\infty} \Psi(x) w(x) d x \lesssim \int_{0}^{\infty}\left|\widehat{f}_{\sin }(t)\right|\left(\int_{0}^{1 / t} w(x) d x\right) d t .
$$


Proof. To apply Lemma 2.3, we must check the convergence conditions on the Fourier transforms assumed there. Denoting both Fourier transforms by $F$, we have for each of the right-hand sides of (2.8) and (2.9)

$$
\int_{0}^{\infty}|F(t)|\left(\int_{0}^{1 / t} w(x) d x\right) d t<\infty .
$$

Further,

$$
\begin{aligned}
& \int_{0}^{u}|F(t)|\left(\int_{0}^{1 / t} w(x) d x\right) d t-\int_{0}^{u}|F(t)|\left(\int_{1 / u}^{1 / t} w(x) d x\right) d t \\
& =\int_{0}^{1 / u} w(x) d x \int_{0}^{u}|F(t)| d t
\end{aligned}
$$

is equivalent to $\int_{0}^{u}|F(t)| d t$ for any fixed $u$. Both integrals on the left-hand side of (2.11) are bounded for any $u$ because of (2.10). Now, Lemma 2.3 yields

$$
\int_{0}^{\infty} \Psi(x) w(x) d x \lesssim \int_{0}^{\infty} w(x) \int_{0}^{1 / x}\left|\widehat{f}_{\cos }(t)\right| d t d x
$$

Then (2.8) and (2.9) follow by Fubini's theorem.

\section{Main results}

In the sequel, we will assume that condition (2.6) is fulfilled, i.e. $\int_{1}^{\infty} \frac{f(t)}{t} d t<\infty$. Define

$$
G M^{1}=\{f: f \geq 0, f \in L(0,1), f \in G M\}
$$

and

$$
G M^{2}=\{f: f \geq 0, t f(t) \in L(0,1), f \in G M\}
$$

\subsection{Two-sided $\left(L_{p}, L_{p}\right)$ inequalities}

The following criteria are the central results of our paper. We denote $w_{p}(x):=$ $x^{-p} w(x)$.

Theorem 3.1. Let $1<p<\infty$.

(A) If $w \in A_{p}$ and $f \in G M^{1}$, then

$$
\int_{0}^{\infty}\left|\widehat{f}_{\cos }(x)\right|^{p} x^{p-2} w(1 / x) d x \asymp \int_{0}^{\infty} w(x) f(x)^{p} d x .
$$


(B) If either $w$ or $w_{p}$ belongs to $A_{p}$ and $f \in G M^{2}$, then

$$
\int_{0}^{\infty}\left|\widehat{f}_{\sin }(x)\right|^{p} x^{p-2} w(1 / x) d x \asymp \int_{0}^{\infty} w(x) f(x)^{p} d x .
$$

Remark 3.2. Let us note that assuming condition (2.6) is essential only in lower estimates (where Lemma 2.3 is used), while for the upper estimates it follows from the corresponding conditions on weights. It is proved in [19, Lemma 2] that $w \in A_{p}$ along with $f \in L_{p, w}$ imply $\int_{0}^{1}|f(t)| d t+\int_{1}^{\infty} t^{-1}|f(t)| d t<\infty$. For $w_{p} \in A_{p}$ along with $f \in L_{p, w}$ this yields $\int_{0}^{1} t|f(t)| d t+\int_{1}^{\infty} t^{-1}|f(t)| d t<\infty$. Further, it is known (see [24]) that for $f \in G M$ condition (2.6) implies $f$ to be of bounded variation away from zero. Along with integrability conditions near the origin, this ensures both existence of the Fourier transforms in the improper sense and their uniform convergence on every compact set away from zero (see [12, Chapter 1]).

In the next theorem, we require additional regularity of the weight $w$ to establish the complete characterization of equivalence $\|\widehat{f}\|_{p, \widetilde{w}} \asymp\|f\|_{p, w}$ in terms of $w$. Recall that if a weight $w$ satisfies $w(x) \asymp w(y)$ for any $y \leq x \leq 2 y$, we write $w \in \Delta_{2}^{2}$.

Theorem 3.3. Let $1 \leq p<\infty$ and $w \in \Delta_{2}^{2}$.

(A) Let $1<p<\infty$ and $w$ satisfy

$$
\frac{1}{\delta} \int_{0}^{\delta} w(x) d x \lesssim w(\delta)
$$

Then (3.1) holds for each $f \in G M^{1}$ if and only if $w \in A_{p}$.

( $\left.\mathrm{A}^{\prime}\right)$ Let $p=1$ and $w$ satisfy (3.3). Then (3.1) holds for each $f \in G M^{1}$ if and only if

$$
\int_{\delta}^{\infty} \frac{w(x)}{x} d x \lesssim w(\delta) .
$$

(B) If $w \in A_{2 p}$, then (3.2) holds for each $f \in G M^{2}$. If $w$ satisfies (3.3), then (3.2) implies $w \in A_{2 p}$.

Remark 3.4. Note that in the proof of $\left(\mathrm{A}^{\prime}\right)$ we do not use the condition $w \in \Delta_{2}^{2}$. Also, any increasing weight (see Theorem A) clearly satisfies condition (3.3). In addition, we note that the assertion of Theorem 3.3 is false without the general monotonicity condition (see [6, Section 4], [20, Theorem 8], [28, Section 5]).

When $w$ is a power weight, Theorem 3.3 furnishes necessary and sufficient conditions for Boas' criterion to hold. Thus, Theorem B is extended as follows. 
Corollary 3.5. Suppose $1 \leq p<\infty$ and $w(x)=x^{\gamma}$; then

(A) relation (3.1) holds for $f \in G M$ if and only if $\gamma \in(-1, p-1)$.

(B) relation (3.2) holds for $f \in G M$ if and only if $\gamma \in(-1,2 p-1)$.

Results for trigonometric series with quasi-monotone and general monotone coefficients similar to (A) were obtained in [2] and [35] respectively. When $p=1$, part "if" is known for monotone functions, see [32] and [11]. For the weight $w(x)=x^{\gamma}$, $\gamma>-1$, we illustrate our results (A) and (B) by the next

Example 3.6. Take $f(t)=e^{-t}$, then $\widehat{f}_{\cos }(x)=1 /\left(1+x^{2}\right)$ and $\widehat{f}_{\sin }(x)=x /(1+$ $\left.x^{2}\right)$. We therefore get that $\|f\|_{p, w}<\infty$ provided $\gamma>-1$. On the other hand, $\left\|\widehat{f}_{\cos }(x)\right\|_{p, \widetilde{w}}<\infty$ provided $-1<\gamma<p-1$ (we remind that $\widetilde{w}(x):=w(1 / x) x^{p-2}$ ), while $\left\|\widehat{f}_{\sin }(x)\right\|_{p, \widetilde{w}}<\infty$ provided $-1<\gamma<2 p-1$.

\subsection{Two-sided $\left(L_{p}, L_{q}, L_{s}\right)$ inequalities}

Theorem 3.1 is a partial case of the next two $\left(L_{p}, L_{q}\right)$ and $\left(L_{q}, L_{s}\right)$ Fourier inequalities. Further, we always suppose that $1<p \leq q \leq s<\infty$. Allied to $w$ is the weight $\widetilde{w}(x)=w(1 / x)^{q / p} x^{q / p^{\prime}-1}$. If we use a weight denoted by $\bar{w}$ (in lower estimates, in fact), this is any weight $\bar{w}$ that relates to $w, p, q$, and $s$ by means of the condition

$$
\sup _{r>0}\left(\int_{0}^{r} \bar{w}(x) d x\right)^{1 / s}\left(\int_{r}^{\infty} \widetilde{w}(1 / x)^{-1 /(q-1)} x^{-2} d x\right)^{1 / q^{\prime}}<\infty .
$$

Recall that $\Phi(t)$ and $\Psi(x)$ are given by (2.1) and (2.7), respectively.

Theorem 3.7. Let $f$ be a non-negative admissible function, integrable near the origin, and let $w^{q / p} \in A_{1+\left(q / p^{\prime}\right)}$ and (3.5) be satisfied. Then

$$
\|\Psi\|_{s, \bar{w}} \lesssim\left\|\widehat{f}_{\cos }\right\|_{q, \widetilde{w}} \lesssim\|\Phi\|_{p, w} .
$$

Theorem 3.8. Let $f$ be a non-negative admissible function, with $t f(t)$ integrable near the origin, and let either $w^{q / p}$ or $w_{p}^{q / p}$ belong to $A_{1+\left(q / p^{\prime}\right)}$, and (3.5) be satisfied. Then

$$
\|\Psi\|_{s, \bar{w}} \lesssim\left\|\widehat{f}_{\sin }\right\|_{q, \widetilde{w}} \lesssim\|\Phi\|_{p, w} .
$$

Comparing the right-hand sides of (3.6) and (3.7) with Theorem A, we arrive at Theorem 1.1 (using Lemma 4.4 below).

For the power weights, Theorems 3.7 and 3.8 can be written as follows. 
Corollary 3.9. Suppose $1<p \leq q \leq s<\infty$ and $w(x)=x^{\gamma}, \widetilde{w}(x)=$ $x^{-\gamma q / p+q-q / p-1}, \bar{w}(x)=x^{(\gamma+1) s / p-1} ;$ then

(A) inequality (3.6) holds if $\gamma \in(-1, p-1)$.

(B) inequality (3.7) holds if $\gamma \in(-1,2 p-1)$.

If additionally, $f \in G M$, then

$$
\begin{array}{ll}
\|f\|_{s, \bar{w}} \lesssim\left\|\widehat{f}_{\cos }\right\|_{q, \widetilde{w}} \lesssim\|f\|_{p, w} \quad \text { holds if } \quad \gamma \in(-1, p-1) \\
\|f\|_{s, \bar{w}} \lesssim\left\|\widehat{f}_{\sin }\right\|_{q, \widetilde{w}} \lesssim\|f\|_{p, w} \quad \text { holds if } \quad \gamma \in(-1,2 p-1) .
\end{array}
$$

In the context of weighted classes, estimates (3.6)-(3.9) refine the previous results of Benedetto et al. [5-7], Heinig [18], Jurkat-Sampson [21, 22], Knopf-Rudnick (see [22, pages 258-259]), Sadosky-Wheeden [28], Sagher [29].

\section{Proofs}

We will first prove upper estimates for the cosine and sine Fourier transforms, under more general conditions on $w$ than those in Theorems 3.7 and 3.8.

Theorem 3.7'. Let $f$ be a non-negative admissible function, integrable near the origin. Let the weights $w, \widetilde{w}$, and $\bar{w}$ satisfy (3.5) along with

$$
\sup _{r}\left(\int_{r}^{\infty} w(x)^{q / p} x^{q / p-q-1} d x\right)^{1 / q}\left(\int_{0}^{r} w(x)^{1 /(1-p)} d x\right)^{(p-1) / p}<\infty
$$

and

$$
\sup _{r}\left(\int_{0}^{r} w(x)^{q / p} x^{q / p-1} d x\right)^{1 / q}\left(\int_{r}^{\infty}\left[x^{p} w(x)\right]^{1 /(1-p)} d x\right)^{(p-1) / p}<\infty .
$$

Then inequality (3.6) holds.

Theorem 3.8'. Let $f$ be a non-negative admissible function, with $t f(t)$ integrable near the origin. Let the weights $w, \widetilde{w}$, and $\bar{w}$ satisfy (3.5) and (4.2) along with

$$
\sup _{r}\left(\int_{r}^{\infty} w(x)^{q / p} x^{q / p-2 q-1} d x\right)^{1 / q}\left(\int_{0}^{r}\left[x^{-p} w(x)\right]^{1 /(1-p)} d x\right)^{(p-1) / p}<\infty .
$$

Then inequality (3.7) holds.

Let us explain the logic of our proofs: Theorems $3.7^{\prime}$ and $3.8^{\prime} \Rightarrow$ Theorems 3.7 and $3.8 \Rightarrow$ Theorem 3.1 and the "if" part of Theorem $3.3(p>1)$. Theorems 3.7, 3.8 and Theorem $\mathrm{A} \Rightarrow$ Theorem 1. Further, Propositions 2.1 and $2.4 \Rightarrow$ Theorem 3.3 in case of $p=1$. Corollary 3.9 follows from Theorems 3.7 and 3.8, except the case $\omega(x)=x^{p-1}$ which follows from Theorem $3.8^{\prime}$.

Before proceeding to the proofs we need certain preliminaries. 


\subsection{Weighted Hardy inequalities.}

We will frequently use the following two weighted Hardy's inequalities (see [13]).

Lemma 4.1. Let $1<\alpha \leq \beta<\infty$. Suppose $u, v \geq 0$ on $(0, \infty)$. Then

$$
\left(\int_{0}^{\infty}\left[u(x) \int_{0}^{x} \psi(t) d t\right]^{\beta} d x\right)^{1 / \beta} \lesssim\left(\int_{0}^{\infty}[\psi(x) v(x)]^{\alpha} d x\right)^{1 / \alpha}
$$

holds for each $\psi \geq 0$ if and only if

$$
\sup _{r>0}\left(\int_{r}^{\infty} u(x)^{\beta} d x\right)^{1 / \beta}\left(\int_{0}^{r} v(x)^{-\alpha /(\alpha-1)} d x\right)^{(\alpha-1) / \alpha}<\infty .
$$

The counterpart

$$
\left(\int_{0}^{\infty}\left[u(x) \int_{x}^{\infty} \psi(t) d t\right]^{\beta} d x\right)^{1 / \beta} \lesssim\left(\int_{0}^{\infty}[\psi(x) v(x)]^{\alpha} d x\right)^{1 / \alpha}
$$

holds for each $\psi \geq 0$ if and only if

$$
\sup _{r>0}\left(\int_{0}^{r} u(x)^{\beta} d x\right)^{1 / \beta}\left(\int_{r}^{\infty} v(x)^{-\alpha /(\alpha-1)} d x\right)^{(\alpha-1) / \alpha}<\infty .
$$

In parallel, we will use Hardy's inequalities for $w$ satisfying Muckenhoupt's condition. Here we continue investigations by Sadosky and Wheeden and will use the following two lemmas from [28] (see Lemmas 1a and 2a). Let a function $\psi$ be non-negative.

Lemma 4.2. Let $1<p \leq q<\infty$ and $w^{q / p} \in A_{1+q / p^{\prime}}$. There holds

$$
\begin{aligned}
& \left(\int_{0}^{\infty}\left[x^{1 / p^{\prime}-1 / q} w(1 / x)^{1 / p} \int_{x}^{\infty} \psi(t) d t\right]^{q} d x\right)^{1 / q} \\
& \lesssim\left(\int_{0}^{\infty} \psi(x)^{p} x^{2 p-2} w(1 / x) d x\right)^{1 / p} .
\end{aligned}
$$

Lemma 4.3. Let $1<p \leq q<\infty$ and $w^{q / p} \in A_{1+q / p^{\prime}}$. There holds

$$
\begin{aligned}
& \left(\int_{0}^{\infty}\left[x^{-1 / p-1 / q} w(1 / x)^{1 / p} \int_{0}^{x} \psi(t) d t\right]^{q} d x\right)^{1 / q} \\
& \lesssim\left(\int_{0}^{\infty} \psi(x)^{p} x^{p-2} w(1 / x) d x\right)^{1 / p} .
\end{aligned}
$$

We will also need the following norm estimate for the average operator $\Phi$. 
Lemma 4.4. Let $w \in A_{p}$ or $w_{p} \in A_{p}$ for $1<p<\infty$. Then $\|\Phi\|_{p, w} \lesssim\|f\|_{p, w}$ for each $f \in G M$.

Proof of Lemma 4.4. Using $f \in G M$, we estimate

$$
\|\Phi\|_{p, w} \lesssim\|\Psi\|_{p, w} \equiv\left(\int_{0}^{\infty} w(x)\left(\int_{x / c}^{c x} \frac{f(t)}{t} d t\right)^{p} d x\right)^{1 / p} \lesssim\|f\|_{p, w} .
$$

The last inequality will be true for $w \in A_{p}$ if we have at once

$$
\left(\int_{0}^{\infty} w(x)\left(\frac{1}{x} \int_{0}^{x} f(t) d t\right)^{p} d x\right)^{1 / p} \lesssim\|f\|_{p, w}
$$

and

$$
\left(\int_{0}^{\infty} w(x)\left(\int_{x}^{\infty} \frac{f(t)}{t} d t\right)^{p} d x\right)^{1 / p} \lesssim\|f\|_{p, w} .
$$

Using Lemmas 4.2 and 4.3 with $p=q$ (changing variables in (4.7) and (4.8)), we arrive at (4.9) and (4.10).

Similarly, if $w_{p} \in A_{p}$, then

$$
\left(\int_{0}^{\infty} w_{p}(x)\left(\frac{1}{x} \int_{0}^{x} t f(t) d t\right)^{p} d x\right)^{1 / p} \lesssim\|f\|_{p, w}
$$

and

$$
\left(\int_{0}^{\infty} w_{p}(x)\left(\int_{x}^{\infty} f(t) d t\right)^{p} d x\right)^{1 / p} \lesssim\|f\|_{p, w},
$$

which gives $\|\Psi\|_{p, w} \lesssim\|f\|_{p, w}$.

\subsection{Proof of Theorem 3.1}

When $1<p<\infty$, it follows from Lemmas 4.3 and 4.1 that $w \in A_{p}$ implies (3.5) with $p=q=s$ and $\bar{w}=w$. Then, by Theorem 3.7 with $p=q=s$, we have

$$
\|\Psi\|_{p, w} \lesssim\left\|\widehat{f}_{\cos }\right\|_{p, \widetilde{w}} \lesssim\|\Phi\|_{p, w} .
$$

Now, statement (A) of Theorem 3.1 follows from property (1.7) of general monotone functions on the one hand, and from Lemma 4.4 on the other hand. The statement (B) is proved in the same way. 


\subsection{Proof of Theorem 3.3.}

First, we will conduct the proof of part (B). Let $w \in A_{2 p}, 1 \leq p<\infty$. This and $w \in \Delta_{2}^{2}$ yield

$$
\begin{aligned}
1 & \gtrsim \delta^{-2 p}\left(\int_{0}^{\delta} w(x) d x\right)\left(\int_{0}^{\delta} w(x)^{\frac{1}{1-2 p}} d x\right)^{2 p-1} \\
& \gtrsim \delta^{-2 p}\left(\int_{0}^{\delta} w(x) d x\right)\left(\delta^{2 p-1} w(\delta)^{-1}\right)
\end{aligned}
$$

which gives (3.3).

Further, by [19, pages 232-233], $w \in A_{2 p}$ implies the well-known AriñoMuckenhoupt condition [1]

$$
\int_{x}^{\infty} \frac{w(t)}{t^{2 p}} d t \leq \frac{C}{x^{2 p}} \int_{0}^{x} w(t) d t
$$

the same relation with $r$ in place of $2 p$ will be recorded as $w \in B_{r}$. Denoting $\int_{0}^{t} w(s) d s=: W(t)$, we derive from this

$$
\int_{x}^{\infty} \frac{W(t)}{t^{2 p+1}} d t \lesssim \frac{W(x)}{x^{2 p}}
$$

The latter inequality and the monotonicity of $W$ immediately give

$$
\frac{W(t)}{t^{2 p}} \lesssim \int_{t}^{\infty} \frac{W(s)}{s^{2 p+1}} d s \lesssim \frac{W(x)}{x^{2 p}}, \quad x<t
$$

Hence for any $c>1$,

$$
\frac{W(c x)}{(c x)^{2 p}} \ln c=\frac{W(c x)}{(c x)^{2 p}} \int_{x}^{c x} \frac{d t}{t} \leq C_{1} \int_{x}^{c x} \frac{W(t)}{t^{2 p+1}} d t \leq C_{2} \frac{W(x)}{x^{2 p}} .
$$

Taking $c>1$ such that $\ln c>C_{2}>1$, we choose $0<\varepsilon<1$ satisfying $\ln c=c^{\varepsilon} C_{2}$. This implies $\frac{W(c x)}{W(x)} \leq c^{2 p-\varepsilon}$. The latter gives

$$
\frac{W(t)}{W(x)} \lesssim\left(\frac{t}{x}\right)^{2 p-\varepsilon}, \quad x<t
$$

Indeed, let $t \in\left(c^{k} x, c^{k+1} x\right)$, where $k$ is non-negative integer. For $k=0$, that is, if $t \in(x, c x)$, (4.13) follows from (4.12). For $k \geq 1$, we have

$$
\frac{W(t)}{W(x)} \leq \frac{W\left(c^{k+1} x\right)}{W(x)} \lesssim c^{k(2 p-\varepsilon)} \lesssim\left(\frac{t}{x}\right)^{2 p-\varepsilon} .
$$


In particular, this implies $W \in \Delta_{2}^{2}$. Then for any $x<t$, using $w, W \in \Delta_{2}^{2}$ and conditions (3.3) and (4.13), we get

$$
\frac{w(t)}{t^{2 p-1-\varepsilon}} \lesssim \frac{\int_{t}^{2 t} w(z) d z}{t^{2 p-\varepsilon}} \lesssim \frac{W(2 t)}{t^{2 p-\varepsilon}} \lesssim \frac{W(t)}{t^{2 p-\varepsilon}} \lesssim \frac{W(x)}{x^{2 p-\varepsilon}} \lesssim \frac{w(x)}{x^{2 p-1-\varepsilon}}
$$

Similar argument, applied to condition (3.3) instead of (4.11) along with $w \in \Delta_{2}^{2}$, gives

$$
\frac{w(t)}{t^{-1+\varepsilon}} \gtrsim \frac{w(x)}{x^{-1+\varepsilon}}, \quad x<t .
$$

With (4.14) and (4.15) in hand, we are going to prove equivalence (3.2). Suppose that $1<p<\infty$. By straightforward calculations, all the inequalities (3.5), (4.2), and $\left(4.1^{\prime}\right)$, with $p=q=s, \bar{w}=w$ and $\widetilde{w}(x)=w(1 / x) x^{p-2}$, follow from (4.14) and (4.15). For instance, let us show (4.2). By (4.15),

$$
I_{1}=\left(\int_{0}^{r} w(x) d x\right)^{1 / p} \lesssim\left(r^{1-\varepsilon} w(r) \int_{0}^{r} x^{\varepsilon-1} d x\right)^{1 / p} \lesssim[r w(r)]^{1 / p}
$$

and

$$
\begin{aligned}
I_{2} & =\left(\int_{r}^{\infty}\left[x^{p} w(x)\right]^{1 /(1-p)} d x\right)^{(p-1) / p} \\
& \lesssim\left(\left[r^{1-\varepsilon} w(r)\right]^{1 /(1-p)} \int_{r}^{\infty}\left[x^{\varepsilon+p-1}\right]^{1 /(1-p)} d x\right)^{(p-1) / p} \lesssim[r w(r)]^{-1 / p} .
\end{aligned}
$$

Thus, $\sup _{r} I_{1} I_{2} \lesssim C$, that is, (4.2) holds.

We are now in a position to use Theorem $3.8^{\prime}$, which gives (3.7). Further, using the general monotonicity of $f$ (see (1.7) and Lemma 4.4), we arrive at

$$
\|f\|_{p, w} \lesssim\|\Psi\|_{p, w} \lesssim\left\|\widehat{f}_{\text {sin }}\right\|_{p, w} \lesssim\|\Phi\|_{p, w} \lesssim\|f\|_{p, w} .
$$

Thus, (3.2) is verified for $1<p<\infty$.

When $p=1$, conditions $w \in B_{2}$ and (3.3) give

$$
t \int_{t}^{\infty} \frac{w(x)}{x^{2}} d x+\frac{1}{t} \int_{0}^{t} w(x) d x \lesssim w(t) .
$$

Therefore, by Propositions 2.1 and 2.4, we get

$$
\begin{aligned}
\|f\|_{1, w} \lesssim\|\Psi\|_{1, w} \lesssim\left\|\widehat{f}_{\sin }\right\|_{1, \widetilde{w}} & \lesssim \int_{0}^{\infty}\left(\int_{x / c}^{c x} \frac{f(t)}{t} d t\right) w(x) d x \\
& \asymp \int_{0}^{\infty} \frac{1}{x}\left(\int_{x / c}^{c x} f(t) w(t) d t\right) d x \asymp\|f\|_{1, w},
\end{aligned}
$$

where we have used $w \in \Delta_{2}^{2}$. Thus (3.2) holds. 
Let us now prove the second part in (B). Assume that (3.2) holds for any $G M$ function. Taking $f(z)=\chi_{(0, t)}(z)$, we estimate

$$
\begin{aligned}
t^{2 p} \int_{t}^{\infty} \frac{w(x)}{x^{2 p}} d x & \lesssim \int_{0}^{1 / t}\left|\int_{0}^{t} z x d z\right|^{p} x^{p-2} w(1 / x) d x \\
& \lesssim \int_{0}^{\infty}\left|\int_{0}^{\infty} f(z) \sin z x d z\right|^{p} x^{p-2} w(1 / x) d x \lesssim \int_{0}^{t} w(x) d x,
\end{aligned}
$$

in other words, $w \in B_{2 p}$. Using the same argument as before (note that (3.3) is assumed), we obtain (4.14) and (4.15). These, in turn, imply $w \in A_{2 p}$ for $1 \leq p<$ $\infty$.

To prove the necessity part in (A) and ( $\left.\mathrm{A}^{\prime}\right)$, we use similar argument as above. In this case, for $f(z)=\chi_{(0, t)}(z)$,

$$
t^{p} \int_{t}^{\infty} \frac{w(x)}{x^{p}} d x \lesssim \int_{0}^{\infty}\left|\int_{0}^{\infty} f(z) \cos z x d z\right|^{p} x^{p-2} w(1 / x) d x \lesssim \int_{0}^{t} w(x) d x,
$$

i.e., $w \in B_{p}, 1 \leq p<\infty$. Then for $p=1$, the latter and condition (3.3) give (3.4).

If $1<p<\infty$, then $w \in B_{p}, \int_{0}^{\delta} w(t) \lesssim \delta w(\delta)$, and $w \in \Delta_{2}^{2}$ imply (4.15) and the condition

$$
\frac{w(t)}{t^{p-1-\varepsilon}} \lesssim \frac{w(x)}{x^{p-1-\varepsilon}}, \quad x<t
$$

in place of (4.14). Then both conditions yield $w \in A_{p}$.

We finally show the sufficiency part in $\left(\mathrm{A}^{\prime}\right)$, since the sufficiency part in $(\mathrm{A})$ is given in Theorem 3.1. Let $p=1$. Under our assumptions,

$$
\int_{t}^{\infty} \frac{w(x)}{x} d x+\frac{1}{t} \int_{0}^{t} w(x) d x \lesssim w(t) .
$$

Therefore, using Propositions 2.1 and 2.4, namely, (2.2) and (2.8), we have

$$
\begin{aligned}
\|\Psi\|_{1, w} & \lesssim \int_{0}^{\infty} \frac{w(1 / x)}{x}\left|\widehat{f}_{\cos }(x)\right| d x \equiv\left\|\widehat{f}_{\cos }\right\|_{1, \widetilde{w}} \\
& \lesssim \int_{0}^{\infty}|\Phi(t)|\left[t^{-1} \int_{0}^{t} w(x) d x+\int_{t}^{\infty} x^{-1} w(x) d x\right] d t \lesssim\|\Phi\|_{1, w} .
\end{aligned}
$$

Since $f$ is general monotone (see (1.6)), we estimate

$$
\begin{aligned}
\|\Phi\|_{1, w} & \lesssim\|\Psi\|_{1, w}=\int_{0}^{\infty}\left(\int_{x / c}^{c x} \frac{f(t)}{t} d t\right) w(x) d x \lesssim \int_{0}^{\infty} \frac{f(t)}{t}\left(\int_{t / c}^{c t} w(x) d x\right) d t \\
& \lesssim \int_{0}^{\infty} f(t)\left[t^{-1} \int_{0}^{t} w(x) d x+\int_{t}^{\infty} x^{-1} w(x) d x\right] d t \lesssim\|f\|_{1, w} .
\end{aligned}
$$

Finally, using (1.7), we arrive at $\|f\|_{1, w} \lesssim\|\Psi\|_{1, w} \lesssim\left\|\widehat{f}_{\cos }\right\|_{1, \widetilde{w}} \lesssim\|f\|_{1, w}$, that is, (3.1) with $p=1$. 


\subsection{Upper estimates in Theorems $3.7^{\prime}, 3.8^{\prime}, 3.7,3.8$ and Theorem 1.1.}

To prove upper estimates of $\|\widehat{f}\|_{q, \widetilde{w}}$, we are going to systematically use Lemma 2.2. In order the right-hand sides of (2.4) and (2.5) to be finite, the boundedness of variation on $[1, \infty)$ is needed, since $\int_{2}^{\infty}|d f(t)| \lesssim \int_{1}^{\infty} \frac{\Phi(t)}{t} d t \lesssim \int_{1}^{\infty}|d f(t)|$. As a matter of fact, this follows from the assumptions of the theorems. Indeed, by Hölder's inequality,

$$
\int_{1}^{\infty}|d f(t)| \lesssim \int_{1 / 2}^{\infty} \frac{\Phi(t)}{t} d t \lesssim\|\Phi\|_{p, w}\left(\int_{1 / 2}^{\infty}\left[t^{p} w(t)\right]^{1 /(1-p)} d t\right)^{1 / p^{\prime}} .
$$

The right-hand side is finite, for functions $f$ so that $\|\Phi\|_{p, w}<\infty$, because of (4.2). So we will use Lemma 2.2 below without indicating the details.

Proof of Theorem 3.7'. Using inequality (2.4) of Lemma 2.2, we have

$$
\begin{aligned}
& \int_{0}^{\infty}\left|\widehat{f}_{\cos }(x)\right|^{q}\left[x^{-1} w\left(x^{-1}\right)\right]^{q / p} x^{q-1} d x \\
& \lesssim \int_{0}^{\infty}\left(\int_{0}^{1 / x} \Phi(t) d t\right)^{q}\left[x^{-1} w\left(x^{-1}\right)\right]^{q / p} x^{q-1} d x \\
& \quad+\int_{0}^{\infty}\left(\int_{1 / x}^{\infty} t^{-1} \Phi(t) d t\right)^{q}\left[x^{-1} w\left(x^{-1}\right)\right]^{q / p} x^{-1} d x=J_{1}+J_{2} .
\end{aligned}
$$

Equivalently,

$$
J_{1}=\int_{0}^{\infty}\left\{\left[x^{-1} w\left(x^{-1}\right)\right]^{1 / p} x^{1-1 / q} \int_{x}^{\infty} t^{-2} \Phi(1 / t) d t\right\}^{q} d x
$$

To prove

$$
\begin{aligned}
J_{1} & \lesssim\left(\int_{0}^{\infty}\left\{x^{-2} \Phi(1 / x) w(1 / x)^{1 / p} x^{2-2 / p}\right\}^{p} d x\right)^{q / p} \\
& =\left(\int_{0}^{\infty} \Phi(x)^{p} w(x) d x\right)^{q / p},
\end{aligned}
$$

we just apply (4.5) and (4.6) with appropriate weights $u$ and $v$, where (4.6) is (4.1) after routine calculations.

Further,

$$
J_{2}=\int_{0}^{\infty}\left\{\left[x^{-1} w\left(x^{-1}\right)\right]^{1 / p} x^{-1 / q} \int_{0}^{x} t^{-1} \Phi(1 / t) d t\right\}^{q} d x
$$


Similarly, applying (4.3) with $u(x)=\left[x^{-1} w\left(x^{-1}\right)\right]^{1 / p} x^{-1 / q}$ and $v(x)=$ $\left[x^{-1} w\left(x^{-1}\right)\right]^{1 / p} x^{1-1 / p}$ under condition (4.4), which is (4.2) for the indicated weights, we obtain

$$
\begin{aligned}
J_{2} & \lesssim\left(\int_{0}^{\infty}\left\{x^{-1} w(1 / x)^{1 / p} x^{1-2 / p} \Phi(1 / x)\right\}^{p} d x\right)^{q / p} \\
& =\left(\int_{0}^{\infty} \Phi(x)^{p} w(x) d x\right)^{q / p} .
\end{aligned}
$$

The proof of the right-hand side inequality in (3.6), i.e., $\left\|\widehat{f}_{\cos }\right\|_{q, \widetilde{w}} \lesssim\|f\|_{p, w}$, is complete.

Proof of Theorem 3.8'. Changing variables, we rewrite the obtained bound (2.5) for the sine transform as

$$
\left|\widehat{f}_{\sin }(x)\right| \lesssim x \int_{x}^{\infty} t^{-3} \Phi(1 / t) d t+x^{-1} \int_{0}^{x} t^{-1} \Phi(1 / t) d t .
$$

With this estimate in hand, we estimate

$$
\begin{aligned}
& \int_{0}^{\infty}\left|\widehat{f}_{\sin }(x)\right|^{q}\left[x^{-1} w\left(x^{-1}\right)\right]^{q / p} x^{q-1} d x \\
& \lesssim \int_{0}^{\infty}\left(\int_{x}^{\infty} t^{-3} \Phi(1 / t) d t\right)^{q}\left[x^{-1} w\left(x^{-1}\right)\right]^{q / p} x^{2 q-1} d x \\
& \quad+\int_{0}^{\infty}\left(\int_{0}^{x} t^{-1} \Phi(1 / t) d t\right)^{q}\left[x^{-1} w\left(x^{-1}\right)\right]^{q / p} x^{-1} d x=I_{1}+I_{2} .
\end{aligned}
$$

We wish to bound $I_{1}$ by

$$
\left(\int_{0}^{\infty}\left\{x^{-3} \Phi(1 / x) w(1 / x)^{1 / p} x^{3-2 / p}\right\}^{p} d x\right)^{q / p}=\left(\int_{0}^{\infty} \Phi(x)^{p} w(x) d x\right)^{q / p}
$$

as the case of (4.5) with $u(x)=\left[x^{-1} w\left(x^{-1}\right)\right]^{1 / p} x^{2-1 / q}$ and $v(x)=$ $x^{3-1 / p}\left[x^{-1} w\left(x^{-1}\right)\right]^{1 / p}$. Applying (4.6) with these weights, we arrive at $\left(4.1^{\prime}\right)$ after routine calculations.

The proof of the estimate $I_{2} \lesssim\|\Phi\|_{p, w}$ is similar to (4.18) and (4.19) for the cosine transform, and it follows from (4.2).

Proofs of Theorems 3.7 and 3.8. To prove the inequality $\left\|\widehat{f}_{\cos }\right\|_{q, \widetilde{w}} \lesssim\|f\|_{p, w}$ in Theorem 3.7, we apply the corresponding Hardy inequalities from Lemmas 4.2 and 4.3 under assumption $w^{q / p} \in A_{1+q / p^{\prime}}$. They, in fact, are (4.5) and (4.3), respectively, with appropriate $u$ and $v$, which leads to (4.1) and (4.2). Finally, Theorem $3.7^{\prime}$ implies the claimed estimate. 
To prove Theorem 3.8 when $w^{q / p} \in A_{1+\left(q / p^{\prime}\right)}$, we first observe that (2.5) in Lemma 2.2 gives

$$
\left|\widehat{f}_{\sin }(x)\right| \lesssim \int_{0}^{1 / x} \Phi(t) d t+x^{-1} \int_{1 / x}^{\infty} t^{-1} \Phi(t) d t
$$

Since (4.1) always implies $\left(4.1^{\prime}\right)$, we can repeat the proof for the cosine case.

Let now $\left[x^{-p} w(x)\right]^{q / p} \in A_{1+\left(q / p^{\prime}\right)}$. By Theorem 3.8', it suffices to prove $\left(4.1^{\prime}\right)$ and (4.2) for the weight $x^{-p} w(x)$. Using Lemmas 4.1 and 4.2 with this weight, we get $\left(4.1^{\prime}\right)$. Further, Lemma 4.3 and (4.4) yield

$$
\sup _{r}\left(\int_{0}^{r}\left[x^{-p} w(x)\right]^{q / p} x^{q / p-1} d x\right)^{1 / q}\left(\int_{r}^{\infty} w(x)^{1 /(1-p)} d x\right)^{(p-1) / p}<\infty .
$$

This gives (4.2), which completes the proof.

Proof of Theorem 1.1. The first part follows from Theorem A. Applying Theorems 3.7 and 3.8, we obtain $\|\widehat{f}\|_{q, \widetilde{w}} \lesssim\|\Phi\|_{p, w}$. Further, since $w^{q / p} \in A_{1+\left(q / p^{\prime}\right)}$ yields $w \in A_{p}$ (see (1.4)), we use Lemma 4.4 to finish the proof: $\|\widehat{f}\|_{q, \widetilde{w}} \lesssim\|\Phi\|_{p, w} \lesssim$ $\|f\|_{p, w}$.

\subsection{General lower estimate.}

Below we prove the lower estimates in Theorems 3.7 and 3.8 (and 3.7', 3.8'), that is, $\|\Psi\|_{s, \bar{w}} \lesssim\left\|\widehat{f}_{\cos }\right\|_{q, \widetilde{w}}$ and $\|\Psi\|_{s, \bar{w}} \lesssim\left\|\widehat{f}_{\text {sin }}\right\|_{q, \widetilde{w}}$ under condition (3.5).

Proof. We can apply Lemma 2.3 to functions with locally integrable Fourier transform. The initial assumption in lower estimates is the boundedness of the $\|F\|_{q}, \tilde{w}$, where by $F$ we denote either $\widehat{f}_{\text {cos }}$ or $\widehat{f}_{\text {sin }}$. Applying Hölder's inequality

$$
\int_{0}^{u}|F(x)| d x \leq\left(\int_{0}^{u} \tilde{w}(x)^{-1 /(q-1)} d x\right)^{(q-1) / q}\|F\|_{q, \tilde{w}},
$$

we derive that the right-hand side is bounded by observing that the integral on the right is exactly the last integral in (3.5) (after substituting $x \rightarrow 1 / x$ and denoting $1 / u=r$ for complete similarity).

Using now Lemma 2.3 and obvious substitution, we arrive, with $F$ the same as above, at

$$
\begin{aligned}
\left(\int_{0}^{\infty} \Psi(x)^{s} \bar{w}(x) d x\right)^{1 / s} & \lesssim\left(\int_{0}^{\infty} \bar{w}(x)\left|\int_{0}^{\pi /(2 c x)} F(t) d t\right|^{s} d x\right)^{1 / s} \\
& =\left(\frac{\pi}{2 c} \int_{0}^{\infty} \bar{w}(\pi /(2 c x)) x^{-2}\left|\int_{0}^{x} F(t) d t\right|^{s} d x\right)^{1 / s} \\
& \lesssim\left(\int_{0}^{\infty}\left[x^{-1} w(1 / x)\right]^{q / p} x^{q(1-1 / q)}|F(x)|^{q} d x\right)^{1 / q} \equiv\|F\|_{q, \widetilde{w}}
\end{aligned}
$$


provided, by (4.3) with $\alpha=q, \beta=s, u(x)^{s}=\bar{w}(\pi /(2 c x)) x^{-2}$, and $v(x)^{q}=$ $\left[x^{-1} w(1 / x)\right]^{q / p} x^{q-1}$,

$\sup _{r>0}\left(\int_{r}^{\infty} \bar{w}(\pi /(2 c x)) x^{-2} d x\right)^{1 / s}\left(\int_{0}^{r}\left[x^{-1} w(1 / x)\right]^{-\frac{q}{p(q-1)}} x^{-1} d x\right)^{(q-1) / q}<\infty$.

For $c$ large enough, the latter can be converted, by routine substitutions, to (3.5).

\section{Remarks on multidimensional generalizations}

The obtained results allow us to derive immediately their versions for the multidimensional $(n>1)$ Fourier transform of a radial function $f(x)=f_{0}(|x|), x \in \mathbb{R}^{n}$. Let $\widehat{f}$ denote its usual Fourier transform on $\mathbb{R}^{n}$. The following result due to Leray (see, e.g., [30, Lemma 25.1']) asserts that when

$$
\int_{0}^{\infty} t^{n-1}(1+t)^{(1-n) / 2}\left|f_{0}(t)\right| d t<\infty
$$

the following relation holds

$$
\hat{f}(x)=2 \pi^{(n-1) / 2} \int_{0}^{\infty} I(t) \cos |x| t d t,
$$

where the fractional integral $I$ is given by

$$
I(t)=\frac{2}{\Gamma\left(\frac{n-1}{2}\right)} \int_{t}^{\infty} s f_{0}(s)\left(s^{2}-t^{2}\right)^{(n-3) / 2} d s .
$$

Supposing the weight $w$ to be radial, $w(x)=w_{0}(|x|)$, we present one analogue of Theorem 3.1 (A).

Theorem 5.1. Let a non-negative radial $f \in L_{1}\left(\mathbb{R}^{n}\right)$. If $w_{0} \in A_{p}\left(\mathbb{R}_{+}\right), 1<p<$ $\infty$, then

$$
\int_{\mathbb{R}^{n}}|\widehat{f}(x)|^{p} w_{0}(1 /|x|)|x|^{p-n-1} d x \asymp \int_{0}^{\infty}|I(t)|^{p} w_{0}(t) d t .
$$

This result is proved by passing to the polar coordinates and applying Theorem 3.1. It is worth mentioning that the condition $f \in L_{1}\left(\mathbb{R}^{n}\right)$ implies (5.1), $I(t) \in L_{1}(0,1)$, and $I(t) / t \in L_{1}(1, \infty)$.

Remark 5.2. We observe that for $n=1$ understanding $I$ formally as $f_{0}$ reduces Theorem 5.1 to Theorem 3.1 (A). In case $n=3$ one can write the Boas-type criterion in terms of $f_{0}$. Indeed, the Fourier transform (if exists) of a radial function in $\mathbb{R}^{3}$ is of the form

$$
\hat{f}(x)=4 \pi|x|^{-1} \int_{0}^{\infty} t f_{0}(t) \sin |x| t d t .
$$


Applying the result for the sine transform (B) to the non-negative function $t f_{0}(t)$, with corresponding assumptions on it, we derive that if either $t^{-p} w_{0}(t)$ or $t^{-2 p} w_{0}(t)$ belongs to $A_{p}\left(\mathbb{R}_{+}\right)$, then

$$
\int_{\mathbb{R}^{3}}|\widehat{f}(x)|^{p} w_{0}(1 /|x|)|x|^{3 p-4} d x \asymp \int_{0}^{\infty} f_{0}(t)^{p} w_{0}(t) d t .
$$

Here we use the fact that $t f_{0}(t) \in G M$ if $f_{0} \in G M$.

\section{References}

[1] M. ARiÑo and B. MUCKenhoupt, Maximal functions on classical Lorentz spaces and Hardy's inequality with weights for nonincreasing functions, Trans. Amer. Math. Soc. 320 (1990), 727-735.

[2] R. ASKey and S. WAIngeR, Integrability theorems for Fourier series, Duke Math. J. 33 (1966), 223-228.

[3] W. Beckner, Pitt's inequality and the uncertainty principle, Proc. Amer. Math. Soc. 123 (1995), 1897-1905.

[4] J. J. BenEDETTO and H. P. Heinig, Weighted Hardy spaces and the Laplace transform, In: "Harmonic Analysis", Proc. Lecture Notes Math., Springer, Vol. 992, 1983, 240-277.

[5] J. J. BENEDETTO and H. P. HEInIG, Fourier transform inequalities with measure weights, Adv. Math. 96 (1992), 194- 225.

[6] J. J. Benedetto, H. P. Heinig and R. Johnson, Fourier inequalities with Ap-weights, In: "General Inequalities", 5 (Oberwolfach, 1986), Vol. 80, Birkhäuser, Basel, 1987, 217 232.

[7] J. J. BenEDETTO and H. P. HeINIG, Weighted Fourier inequalities: new proofs and generalizations, J. Fourier Anal. Appl. 9 (2003), 1-37.

[8] J. J. BenEDETTO and J. D. LAKEY, The definition of the Fourier transform for weighted inequalities, J. Funct. Anal. 120 (1994), 403-439.

[9] S. BLOOM and G. SAMPSON, Weighted spherical restriction theorems for the Fourier transform, Illinois J. Math. 36 (1992), 73-101.

[10] R. P. BOAS, The integrability class of the sine transform of a monotonic function, Studia Math. 44 (1972), 365-369.

[11] R. P. BoAs, "Integrability Theorems for Trigonometric Transforms", Springer-Verlag, New York, 1967.

[12] S. BochneR, "Lectures on Fourier Integrals", Princeton University Press, Princeton, N.J., 1959.

[13] J. S. BRADley, Hardy inequalities with mixed norms, Canad. Math. Bull. 21 (1978), 405408.

[14] J. CERDÀ and J. MARTín, Weighted Hardy inequalities and Hardy transforms of weights, Studia Math. 139 (2000), 189-196.

[15] D. CRUZ-URIBE, Piecewise monotonic doubling measures, Rocky Mountain J. Math. 26 (1996), 545-583.

[16] M. I. D'YACHENKO and E. D. NuRsulTANOV, The Hardy-Littlewood theorem for trigonometric series with $\alpha$-monotone coefficients, Mat. Sb. 200 (2009), 45-60.

[17] M. L. Gol'DMAN, Estimates for multiple Fourier transforms of radially symmetric monotone functions, Sib. Math. J. 18 (1978), 391-406.

[18] H. P. HEINIG, Weighted norm inequalities for classes of operators, Indiana Univ. Math. J. 33 (1984), 573-582. 
[19] R. Hunt, B. MucKenhoupt and R. WheEden, Weighted norm inequalities for the conjugate function and Hilbert transform, Trans. Amer. Math. Soc. 176 (1973), 227-251.

[20] R. JoHnson, Recent Results on Weighted Inequalities for the Fourier Transform, In: "Seminar Analysis of the Karl-Weierstrass-Institute of Mathematics", 1986/87 (Berlin, 1986/87), Teubner-Texte Math., Vol. 106, Leipzig, 1988, 287-296.

[21] W. B. JURKAT and G. SAMPSON, On maximal rearrangement inequalities for the Fourier transform, Trans. Amer. Math. Soc. 282 (1984), 625-643.

[22] W. B. JURKAT and G. SAMPSON, On rearrangement and weight inequalities for the Fourier transform, Indiana Univ. Math. J. 33 (1984), 257-270.

[23] E. LIFLyAND and S. TIKHONOV, Extended solution of Boas' conjecture on Fourier transforms, C.R. Acad. Sci. Paris, Ser.1 346 (2008), 1137-1142.

[24] E. LIFLYAND and S. TIKHONOV, The Fourier Transforms of General Monotone Functions, In: "Analysis and Mathematical Physics", Trends in Mathematics, Birkhäuser, 2009, 373391.

[25] E. LifLyAND and S. TIKHONOV, A concept of general monotonicity and applications, Math. Nachr. 284 (2011), 1083-1098.

[26] B. Muckenhoupt, Weighted norm inequalities for the Fourier transform, Trans. Amer. Math. Soc. 276 (1983), 729-742.

[27] H. R. PITT, Theorems on Fourier series and power series, Duke Math. J. 3 (1937), 747-755.

[28] C. SADOSKY and R. L. WHEEDEN, Some weighted norm inequalities for the Fourier transform of functions with vanishing moments, Trans. Amer. Math. Soc. 300 (1987), 521-533.

[29] Y. SAGHeR, Integrability conditions for the Fourier transform, J. Math. Anal. Appl. 54 (1976), 151-156.

[30] S. G. SAmko, A. A. Kilbas and O. I. Marichev, "Fractional Integrals and Derivatives: Theory and Applications", New York, NY: Gordon and Breach, 1993.

[31] J.-O. STRÖMBERG and R. L. WHEEDEN, Weighted norm estimates for the Fourier transform with a pair of weights, Trans. Amer. Math. Soc. 318 (1990), 355-372.

[32] B. SZ.-NAGY, Séries et intégrales de Fourier des fonctions monotones non bornées, Acta Sci. Math. (Szeged) 13 (1949), 118-135.

[33] E. M. STEIN, Interpolation of linear operators, Trans. Amer. Math. Soc. 83 (1956), 482492.

[34] E. M. SteIn, "Harmonic Analysis, Real Variable Methods, Orthogonality, and Oscillatory Integrals", Princeton Univ. Press, Princeton, NJ, 1993.

[35] S. TIKHONOV, Trigonometric series with general monotone coefficients, J. Math. Anal. Appl. 326 (2007), 721-735.

[36] E. C. Titchmarsh, "Introduction to the Theory of Fourier Integrals", Oxford, 1937.

Department of Mathematics

Bar-Ilan University

52900 Ramat-Gan, Israel

liflyand@math.biu.ac.il

ICREA and Centre de Recerca Matemàtica Apartat 5008193 Bellaterra

Barcelona, Spain

stikhonov@crm.cat 